\title{
Serum Albumin as a Cross-Reactive Component in Furry Animals May Be Related to the Allergic Symptoms of Patients with Rhinitis
}

\author{
Zhifeng Huang (iD* \\ Huiqing Zhu (iD* \\ Runpei Lin \\ Liting Wu \\ Nairui An \\ Peiyan Zheng \\ Baoqing Sun (D)
}

National Center for Respiratory Medicine, The First Affiliated Hospital of Guangzhou Medical University, National Clinical Research Center for Respiratory Disease, State Key Laboratory of Respiratory Disease, Guangzhou Institute of Respiratory Health, First Affiliated Hospital of Guangzhou Medical University, Guangzhou, 510120, People's Republic of China

*These authors contributed equally to this work
Correspondence: Baoqing Sun; Peiyan

Zheng

National Center for Respiratory

Medicine, The First Affiliated Hospital of

Guangzhou Medical University, National

Clinical Research Center for Respiratory

Disease, State Key Laboratory of

Respiratory Disease, Guangzhou Institute of Respiratory Health, First Affiliated

Hospital of Guangzhou Medical

University, 15I Yan Jiang Road,

Guangzhou, 510120, People's Republic of China

Tel +86 I88I678I502

Email sunbaoqing@vip.163.com;

gdmcslxx@126.com
Background: The prevalence of allergies has increased significantly in the past decade. Further research on allergic diseases caused by furry animals is of great importance for the clinical prevention, diagnosis and treatment of allergies.

Objective: To identify the sensitization profile and clinical association of various furry animal crude extracts and components based on component resolved diagnosis (CRD).

Methods: A total of 211 patients with allergic rhinitis with sensitivities to cats and/or dogs were recruited, and the specific immunoglobulin E (sIgE) against various furry animals (such as dog/cat extracts and their components, pigeon, parrot, duck, chicken, sheep, rat, mouse, goose, cow and horse extracts) were measured to analyze the sensitization profiles, crossreactivity and clinical relevance with regards to allergies.

Results: A total of $91.67 \%$ of cat-sensitized patients were sensitive to Fel d 1, while only $16.03 \%$ of cat-sensitized patients responded to Fel d 2. Can f 1 and Can $\mathrm{f} 5$ were the major components of dogs, and the positive rates were $23.53 \%$ and $16.18 \%$, respectively. Twenty percent of patients were sensitized to 10 other furry animals, and the positive rate was between $0 \%$ and $19.12 \%$. There was a significant correlation between components (Can f 1-5 and Fel d 2) and 5 furry animals (mouse, sheep, Horse, rat, cow), especially between serum albumin (SA) (Can f 3, Fel d 2) and furry animals. Most of the animal crude extracts and components sensitization rates in patients who were SA-positive were significantly higher than that of patients who were SA-negative. In particular, for sensitization to mice, sheep, horses, rats and cows, more than 10-fold higher in patients who were SA-positive than in patients who were SA-negative. The VAS of symptoms and life of quality (LoQ) in the SA-sensitized patients was higher than that in unsensitized patients, and the patients with lipocalin sensitivities had a worse LoQ.

Conclusion: Serum albumin Fel d 2 and Can $\mathrm{f} \mathrm{3,} \mathrm{as} \mathrm{minor} \mathrm{allergens} \mathrm{in} \mathrm{cats} \mathrm{and} \mathrm{dogs,} \mathrm{but}$ not lipocalin or prostatic kallikrein, is associated with other furry animals presumably due to serum albumin cross-reactivity. Patients sensitized with serum albumin had a significantly higher risk of sensitization to other animals and had a higher rhinitis VAS score.

Keywords: serum albumin, furry animals, allergy rhinitis, component-resolve diagnosis, cross sensitization

\section{Introduction}

Over the past three to four decades, the morbidity of allergic diseases such as allergic rhinitis (AR) and asthma (AS) has increased globally, and allergic diseases have become a serious global health problem. Furry animals are major sources of indoor allergens and have been widely studied as a major risk factor for airway 
allergic diseases. ${ }^{1-3}$ With the increasing frequency of pet ownership (especially cats and dogs), the risk of allergies is also increasing. ${ }^{4}$ More than half of the global population have a pet in their home. An international study from 22 countries showed that in China, $25 \%$ of people own a dog and $10 \%$ of people own a cat in their household. ${ }^{5}$ Some studies found that individuals who are sensitized to cats and dogs have a higher risk of developing allergies to other furry animals, ${ }^{6}$ and this may aggravate allergic airway hyper-responsiveness and airway inflammation, lowering quality of life. ${ }^{7}$

The routine diagnosis of allergies to furry animals based on crude extracts is usually not sufficient to explain the poly-sensitization and clinical correlation. Component resolved diagnosis (CRD) allows for identification of cross-reactions and the investigation of clinical response at the molecular level. ${ }^{8}$ Immunoglobulin E (IgE)-mediated chronic airway allergic diseases caused by furry animals are becoming increasingly common. Many patients may also be sensitive to more than one furry animal; therefore, the detection of the components of furry animals is helpful in evaluating the clinical relevance and identifying risk markers for allergic symptoms. ${ }^{9}$

Allergen molecules in cats (Felis domesticus) and dogs (Canis familiaris) have previously been widely described. The lipocalin protein family is the most important aeroallergen in furry animals. Although some lipocalin allergens share only $18-25 \%$ sequence identity, their 3-Dimensional structures are highly similar. ${ }^{10}$ It was reported that there is a cross-reaction among furry animal lipocalin proteins (such as Can $\mathrm{f} 1$ and 2, Fel $\mathrm{d} 7$ and 4, Equ c 1, Mus $\mathrm{m} 1$ and Bos d 2). ${ }^{11-13}$ Some furry animals` serum albumin (SA), such as Can f 3 (dog), Fel d 2 (cat), Bos d 6 (cow), Equ c 3 (horse), Cav p 4 (guinea pig), Sus s 1 (pig) and Gal d 5 (chicken), have been well identified and officially confirmed by the International Union of Immunological Societies (IUIS) allergen nomenclature subcommittee [http://www.allergen.org]. ${ }^{14}$ To verify the crossreactivity between $\mathrm{SA}$, the results of previous studies have demonstrated that SA in rats, mice, chickens and other animals can induce the release of histamine from basophils in patients with sensitivities to dog SA, and approximately $30 \%$ of patients who are allergic to cats or dogs can react with albumin from different furry animals (such as pig, cattle, horse, guinea pig, rabbit, etc). ${ }^{15,16}$ Can $\mathrm{f} 5$, a prostatic kallikrein (PK) expressed in male dog prostates, has been identified as another major dog allergen. ${ }^{17}$

Although most people are tolerant to SA in mammals, there are still a few people who develop severe allergic reactions to $\mathrm{SA}$. It has been reported for decades that there is an IgE cross-reaction among the SA of dogs, cats, horses, mice and cattle, which is associated with the clinical symptoms and lung function of patients. ${ }^{16,18-20} \mathrm{At}$ present, there have been some studies about allergic respiratory diseases caused by SA from cat and dog hair and dander. However, little research has focused on the cross-reaction and co-sensitization among furry animals (such as sheep, rats, mice, cows, horses, etc.) and the effects of this cross-reactions on the clinical symptoms of sensitized patients.

In the current study, 211 patients with AR and/or AS were enrolled to evaluate their visual analog scale (VAS) scores for symptoms and life of quality (LoQ). The specific IgE (sIgE) levels against various furry animal proteins (such as dog/cat extracts and their components, and pigeon, parrot, duck, chicken, sheep, rat, mouse, goose, cow and horse extracts) were measured. We analyzed the sensitization profiles, cross-reactions and impact of AR in patients to provide reasonable recommendations for the prevention and clinical diagnosis of furry animal allergies.

\section{Materials and Methods Subjects}

This study was conducted from January 2017 to December 2019 at the Department of Allergy and Clinical Immunology and the Department of Pediatrics at the First Affiliated Hospital of Guangzhou Medical University. Subjects who fulfilled all of the following criteria were enrolled: 1) AR with or without AS confirmed by experienced clinicians according to the guidelines of Allergic Rhinitis and its Impact on Asthma (ARIA) ${ }^{21}$ and the Global Initiative for Asthma guidelines (GINA) (http:// ginasthma.org/gina-reports); 2) currently have symptoms related to rhinitis; 3) have sensitivities to cat and/or dog allergens as screened and confirmed by the EUROLINE system ( $\operatorname{sgE} \geq 0.35 \mathrm{IU} / \mathrm{mL}) ; 4)$ all ages and genders were enrolled; 5) have been informed of the research purpose and voluntarily signed the informed consent form. The exclusion criteria were (1) history of specific allergen immunotherapy, (2) upper respiratory infection or chronic rhinosinusitis, (3) the use of concomitant medications (eg, antihistamines, intranasal corticosteroids) that could affect AR symptoms within 2 weeks before enrollment, and (4) pregnancy or lactation. The flow chart of this study is shown in Figure 1. A total of 211 patients with AR with or without AS were included. According to the sensitization of cat and 


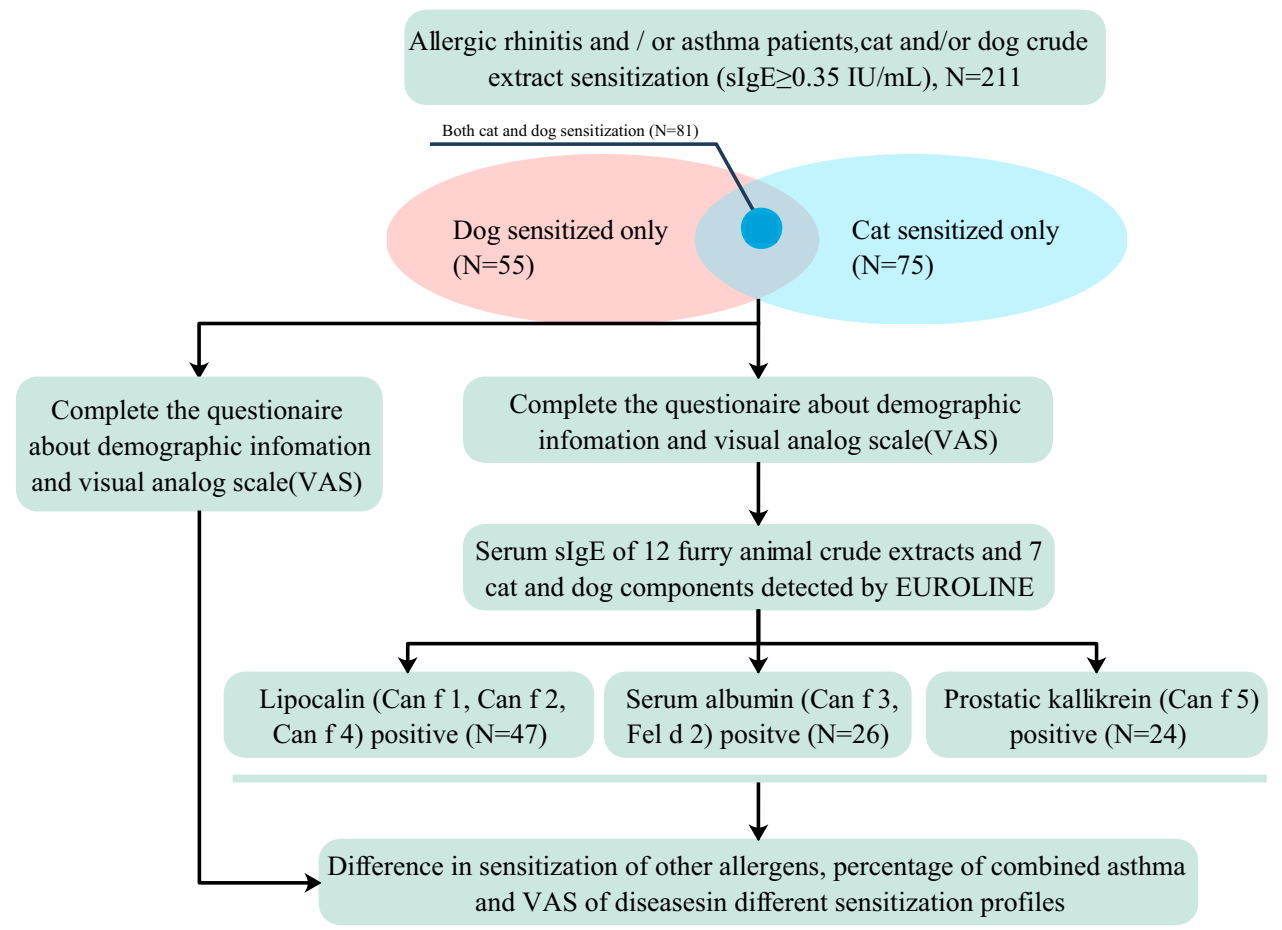

Figure I Research cohort recruitment flowchart. Patients who were clinically confirmed to have allergic rhinitis were screened for cat and dog allergen sensitivities, and a questionnaire about demographic information and visual analog scores (VASs) was given to each patient. After obtaining informed consent from all patients, serum samples from patients were used to further examine the sensitization profiles to common furry animals and cat/dog components.

Abbreviations: Can f, Canis familiaris; Fel d, Felis domesticus.

dog allergens, the patients were divided into cat-sensitized only $(\mathrm{N}=75)$, dog-sensitized only $(\mathrm{N}=55)$ and both cat and dog double-sensitized $(\mathrm{N}=81)$. Five milliliters of venous blood was collected from each patient, the blood was centrifuged at $1000 \mathrm{xg}$ for $10 \mathrm{~min}$, and the serum was stored at $-80^{\circ} \mathrm{C}$ until use.

\section{Questionnaire}

The VAS was proposed by the Joint Task Force on Practice Parameters and is a simple quantitative measure largely used to assess the symptom severity and LoQ of patients with $\mathrm{AR}^{22}$ A VAS score of $0-10$ was used to evaluate nasal (sneezing, rhinorrhea, nasal congestion, nasal itching, and loss of sense of smell) and ocular (eye itching, conjunctival redness, watery eyes, and eyelid edema) symptoms and LoQ (impact on sleep, impact on work life, impact on social life, and physical activities). A response of 0 was "no symptoms", and 10 was "very severe symptoms". Since we cannot confirm whether the patient's nose symptoms are caused by furry animals through the provocation test (the local ethics committee forbids Allergen Challenge Test), we can only score the patients' existing rhinitis symptoms without exploring whether these symptoms are caused by furry animals

\section{Measurement of Allergen-slgE}

SIgEs (major recombinant and natural extract) for the following allergen sources was measured by EUROLINE (EUROIMMUN AG, Lübeck) according to the manufacturer's instructions: dog, cat, pigeon, parrot, duck, chicken, sheep, rat, mouse, goose, cow, and horse. SIgEs against cat and dog major allergen components were measured and included the allergen components Can f 1, Can f 2, Can f 3, Can f 4, Can f 5 and Fel d 1, Fel d 2. sIgE levels were expressed in international units per milliliter $(\mathrm{IU} / \mathrm{mL})$ with the following range: $0.35-100 \mathrm{IU} / \mathrm{mL}$. Tests with sIgE level less than $0.35 \mathrm{IU} / \mathrm{mL}$ were defined as sIgE-negative, and tests with $\operatorname{sgE}$ levels greater than or equal to $0.35 \mathrm{IU} / \mathrm{mL}$ were defined as sIgE-positive. SIgE-positive tests were categorized into the following 6 classes: class $1(\geq 0.35$ to $<0.70$ $\mathrm{IU} / \mathrm{mL}$ ), class $2(\geq 0.70$ to $<3.50 \mathrm{IU} / \mathrm{mL}$ ), class 3 ( $\geq 3.50$ to $<17.50 \mathrm{IU} / \mathrm{mL})$, class $4(\geq 17.50$ to $<50 \mathrm{IU} / \mathrm{mL})$, class $5(\geq$ 50 to $<100 \mathrm{IU} / \mathrm{mL})$, and class $6(\geq 100 \mathrm{IU} / \mathrm{mL})$.

\section{Statistical Analysis}

Categorical data are reported as percentages which show the proportion of positive sIgE patients. Proportions were compared between the groups using the chi-squared test $\left(\chi^{2}\right)$, and the F-test was used to compare the variance of data 
among the groups. Parametric quantitative data are presented as the mean \pm standard deviation, and nonparametric quantitative data are presented as the median (interquartile range). The $t$-test or Mann-Whitney $U$-test was used to compare the distribution differences between the two groups, and a one-way ANOVA or Kruskal-Wallis $H$-test was used to compare the distribution differences between multiple groups. A hierarchical cluster test was used to classify all variables by analyzing the similarity or dissimilarity of the data, and an optimal scale analysis was used to verify the correlation between allergen components. A Venn diagram was used to show the cross-sensitizations among multiple allergens. Statistical analyses were performed using Excel 2016 (Microsoft $^{\circledR}$ Excel $^{\circledR}$ 2016) and Statistical Package for the Social Sciences 22.0 (International Business Machines Corporation Corp., Armonk, NY). All $\mathrm{p}$ values were based on two-sided tests and were considered statistically significant at $P<0.05$.

\section{Results}

\section{Characteristics of the Study Subjects}

In the included patients, $85(40.28 \%)$ were males and the median age was $14(6,24)$ years (median (IQR)). The ratio of adult to non-adult patients was $0.49(69 / 142)$. There were 70 patients with AR and 141 patients with AR \& AS. The VAS scores of symptoms and LoQ were 3.56 (3.00, $4.28)$ and $5.25(4.25,5.75)$, respectively. In addition, the patients were divided into dog-sensitized only $(\mathrm{N}=55)$, catsensitized only $(\mathrm{N}=75)$, dog and cat double-sensitized $(\mathrm{N}=81)$ according to the sensitization of the patients to cat and dog extracts. There were no statistically significant differences in sex, age, asthma prevalence among the three groups (all $P>0.05$ ) (Table 1). On the other hand, the rhinitis symptom score of dog and cat double-sensitized patients was significantly higher than that of dogsensitized only and cat-sensitized only patients (3.33 $(2.56,4.00)$ vs $3.44(2.89,3.94)$ vs 4.00 (3.33, 4.56), $P<0.01)$, and the LoQ score of $\operatorname{dog}$ and cat doublesensitized patients was significantly higher than that of dog-sensitized only patients. $(4.50(3.25,5.50)$ vs 5.50 (4.75, 6.00), $P<0.01)$.

\section{Sensitization Profile of Furry Animal Components and Crude Extracts}

Among the dog-sensitized patients, Can $\mathrm{f} 1$ had the highest positive rate of $23.53 \%$, followed by Can $\mathrm{f} 5$ (16.18\%),

Table I Demographic Characteristics of the Participants

\begin{tabular}{|c|c|c|c|c|c|}
\hline & Total Cohort & $\begin{array}{c}\text { Dog-Sensitized } \\
\text { Only }\end{array}$ & $\begin{array}{c}\text { Cat-Sensitized } \\
\text { Only }\end{array}$ & $\begin{array}{c}\text { Dog and Cat Double- } \\
\text { Sensitized }\end{array}$ & P value* \\
\hline $\mathbf{N}$ & 211 & 55 & 75 & 81 & - \\
\hline \multicolumn{6}{|l|}{ Sex, n (\%) } \\
\hline Male & $85(40.28)$ & $35(63.64)$ & $43(57.33)$ & $48(59.26)$ & 0.765 \\
\hline Female & $126(59.72)$ & $20(36.36)$ & $32(42.67)$ & $33(40.74)$ & \\
\hline \multicolumn{6}{|l|}{ Age, n (\%) } \\
\hline Median (IQR) & $14(6,24)$ & $10(6,18)$ & $13(9,24)$ & $9(5,26)$ & 0.062 \\
\hline$<18$ years & $142(67.30)$ & $4 \mid(74.55)$ & $46(6 I .33)$ & $55(67.90)$ & 0.281 \\
\hline$\geq 18$ years & $69(32.70)$ & $14(25.44)$ & $29(38.67)$ & $26(32.10)$ & \\
\hline \multicolumn{6}{|l|}{ Diagnosis, n (\%) } \\
\hline Allergy rhinitis & $70(40.28)$ & 17 (30.9I) & $23(30.67)$ & $30(37.04)$ & 0.642 \\
\hline $\begin{array}{l}\text { Allergy rhinitis with } \\
\text { asthma }\end{array}$ & $14 \mid(66.82)$ & $38(69.09)$ & $52(69.33)$ & $51(62.96)$ & \\
\hline \multicolumn{6}{|l|}{$\begin{array}{l}\text { VAS score, median } \\
\text { (IQR) }\end{array}$} \\
\hline Symptom & $3.56(3.00,4.28)$ & $3.33(2.56,4.00)$ & $3.44(2.89,3.94)$ & $4.00(3.33,4.56)$ & $<0.001$ \\
\hline Life of quality & $5.25(4.25,5.75)$ & $4.50(3.25,5.50)$ & $5.25(4.38,5.75)$ & $5.50(4.75,6.00)$ & $<0.001$ \\
\hline
\end{tabular}

Notes: Proportions were compared between the groups using chi-squared test $\left(\chi^{2}\right)$, and for nonparametric quantitative data, the Kruskal-Wallis $H$-test was used for comparison between groups. *P value refers to the comparison among the three groups of dog-sensitized only, cat-sensitized only, dog and cat double-sensitized. If the difference between groups is statistically significant, use bold fonts.

Abbreviation: VAS, visual analog scale. 
and Can f 4, Can f 2 and Can $\mathrm{f} 3$ all had the same positive rate of $11.76 \%$. The positive rates of Fel $\mathrm{d} 1$ and Fel $\mathrm{d} 2$ in cat-positive samples were $91.67 \%$ and $16.03 \%$, respectively. The positive rate of Can $\mathrm{f} 1,2,4$ and 5 in dogsensitized only and double-sensitized patients was significantly higher than that in cat-sensitized patients, while the positive rate of Fel $\mathrm{d} 1$ in cat-sensitized and doublesensitized patients was higher than that in dog-sensitized patients $(P<0.05)$. In addition, we noticed that the positive rate of SA (Can f 3 and Fel d 2) in patients with doublesensitized was significantly higher than that in dog- or catsensitized only patients $(P<0.01)$ (Figure $2 \mathrm{~A}$ ).

For chicken, sheep, rat, mouse, cow, horse extracts, the positive rates in double-sensitized patients were significantly higher than that of dog- or cat-sensitized only patients. Their positive rates in double-sensitized patients were cow $(27.16 \%)>$ sheep $(23.46 \%)>$ Rat $(20.99 \%)>$ mouse $(19.75 \%)>$ horse $(16.05 \%)>$ chicken $(7.41 \%)$, while their positive rates were all low (all $<8 \%$ ) in dog-or catSensitized only patients (Figure $2 B$ ). In addition, the positive rate of parrot and duck in the three groups was all $0 \%$, and pigeon and goose were detected positive samples only in dog-sensitized patients, $3.64 \%$ and $1.82 \%$ respectively.

\section{Analysis of Potential Associations with Multiple Allergens}

Because the crude extracts of duck and parrot were all negative in all 211 patients enrolled, these allergens were excluded from follow-up analysis. Hierarchical cluster and optimal scale analyses were performed for the 10 furry animal extracts and the 7 components of cats and dogs to explore potential relationships. The results of hierarchical cluster analysis (Figure 3A) showed that there were strong potential relationships among Can $\mathrm{f} 3$, mouse, sheep, Fel d 2 and horse, rat, and cow allergens. Moreover, the results of optimal scale analysis (Figure 3B) also showed that all components of dogs (Can f 1, Can f 2, Can f 3, Can f 4, Can $\mathrm{f} \mathrm{5)}$ and Feld 2 were potentially associated with furry animals such as mice, sheep, horses, rats and cows.

\section{Plot the Venn Diagram}

Based on the above results of hierarchical cluster and optimal scale analyses, we hypothesized that the 5 furry animals (mouse, sheep, horse, rat, cow) cross-reacted with the components of cat/dog, and we plotted the Venn diagram. First, we analyzed the positive crossover of Can $\mathrm{f} 3$ and Fel d 2 (Figure 4A) because they all belong to SA, and
$18(69.2 \%)$ cases reacted to both Can f 3 and Fel d 2. For lipocalin (Can f 1, Can f 2, Can f 4), 7 (14.9\%) cases were positive for all three components, and 19 (40.4\%) cases reacted to two or more components (Figure 4B). There was no obvious cross-reaction between SA, lipocalin and PK because $64.6 \%$ of sera were monovalently sensitized to one of these three proteins (Figure 4C).

Moreover, we plotted the Venn diagram to reflect the intersection and union of the extracts from 5 furry animals and one of the three proteins (Figure 4D-F). There was considerable overlap in $\mathrm{IgE}$ binding to SA, with $48.5 \%$ of sera reacting to one or more of the 5 furry animal extracts and $36.4 \%$ of sera that were cross-sensitized by SA and all 5 furry animal extracts. However, for lipocalin and PK, only 13.8\% and $5.7 \%$ of sera overlapped with the extracts from 5 furry animals.

\section{Sensitization Profile Based on the Above Three Proteins}

Furthermore, we divided the patients into subgroups according to whether one of the above three proteins was sensitized, and analyzed the sensitization rates of other crude extracts and components (Table 2). In the patients who were SA-positive, the sensitization rate of most of the animal crude extracts and components was significantly higher than that of the patients who were SA-negative, except for can $\mathrm{f} 5$, pigeon and goose. It is worth noting that the OR values of mice, sheep, horses, rats and cows were all more than 10 .

Compared with lipocalin-negative patients, allergens such as Can f, SA, PK, sheep, rat, mouse, and horse had significantly higher sensitization rates in positive patients, and their OR values ranged from 1.35 to 7.56 . The sensitization rates of lipocalin, sheep, mice, and horses were higher in PK-positive patients $(P<0.05)$, with $\mathrm{OR}$ value ranging from 2.60 to 4.68 .

\section{Clinical Characteristics Based on the Above Three Proteins}

A higher positive rate was observed in patients sensitized to three proteins, especially SA, and we investigate the association between these proteins and the clinical characteristics of the patients. The proportion of asthma among the $211 \mathrm{AR}$ patients enrolled in this study ranged between $60 \%$ and $80 \%$, regardless of whether patients were sensitized to SA, lipocalin, or PK protein, and there was no significant difference between the positive group and the negative group (all $P>$ 0.05). (Data not displayed). 


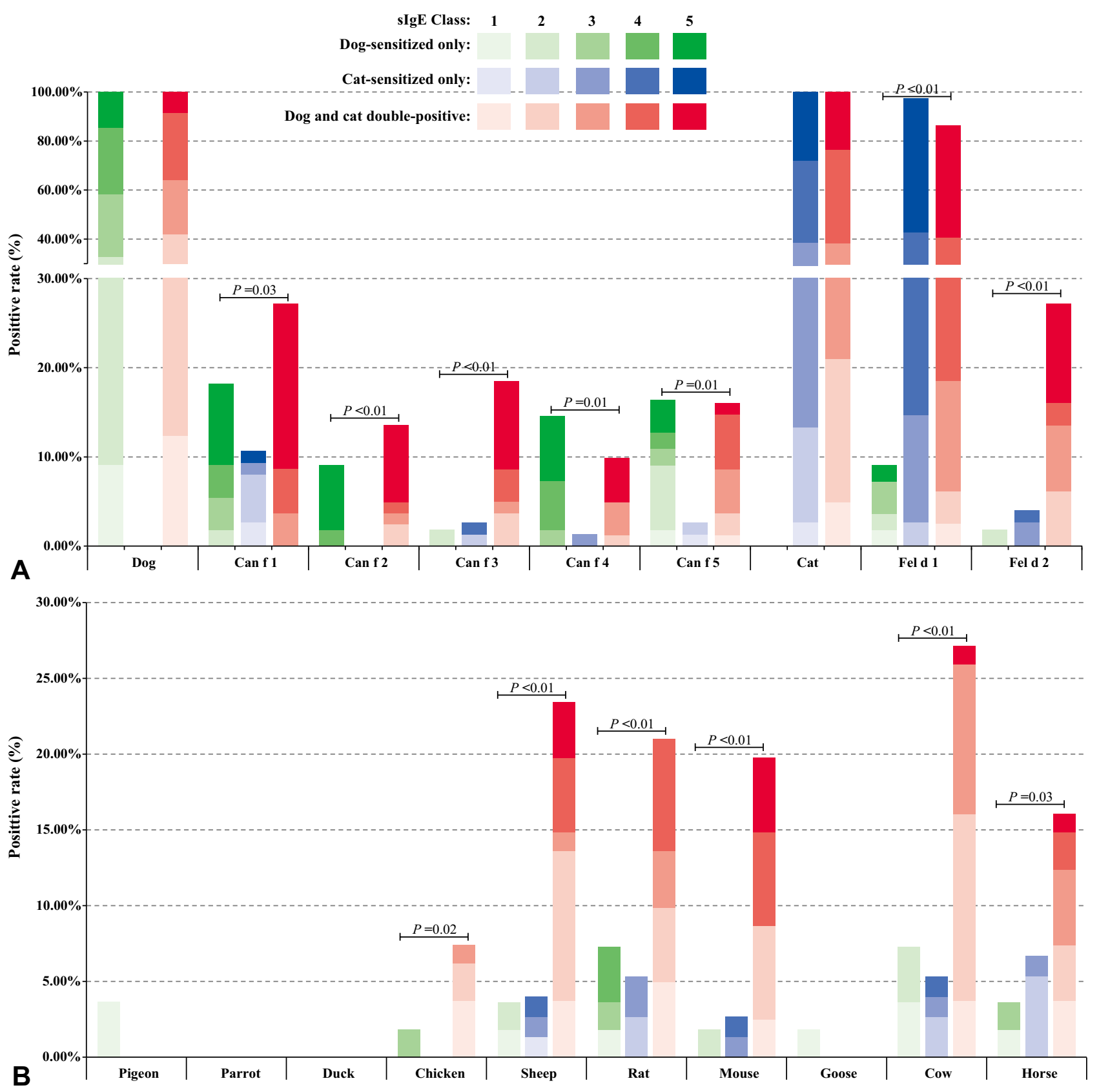

Figure 2 Distribution of positive rates of the components (A) and other furry animal crude extracts (B) in dog-sensitized only, cat-sensitized only and both dog and cat double-sensitized patients. Kruskal-Wallis $\mathrm{H}$-test was used to compare the proportion differences between multiple groups. The color shades refers to the percentage of slgE class I-5.

Abbreviations: Can f, Canis familiaris; Fel d, Felis domesticus.

However, the VAS score of symptoms in the SAsensitized patients was $4.17(3.56,4.78)$, which was higher than that $(3.44(2.89,4.22))$ in unsensitized patients (MannWhitney $\mathrm{U}=1245, P<0.001$ ) (Figure $5 \mathrm{~A}$ ). Meanwhile, the VAS of LoQ was also higher in SA-sensitized patients (5.75 $(5.50,6.50)$ vs $5.00(4.00,5.75), P<0.01)$ (Figure $5 \mathrm{~B})$. There was no significant difference in the VAS of symptoms between the lipocalin-positive and lipocalin-negative patient groups, but the LoQ score was higher in the sensitized patients $(5.75(5.00,6.00)$ vs $5.00(4.00,5.75), P<0.01)$. There was no significant difference in VAS scores for either symptoms or LoQ for PK proteins.

\section{Discussion}

The prevalence of allergies has increased significantly in the past decade. Allergies caused by furry animals, 


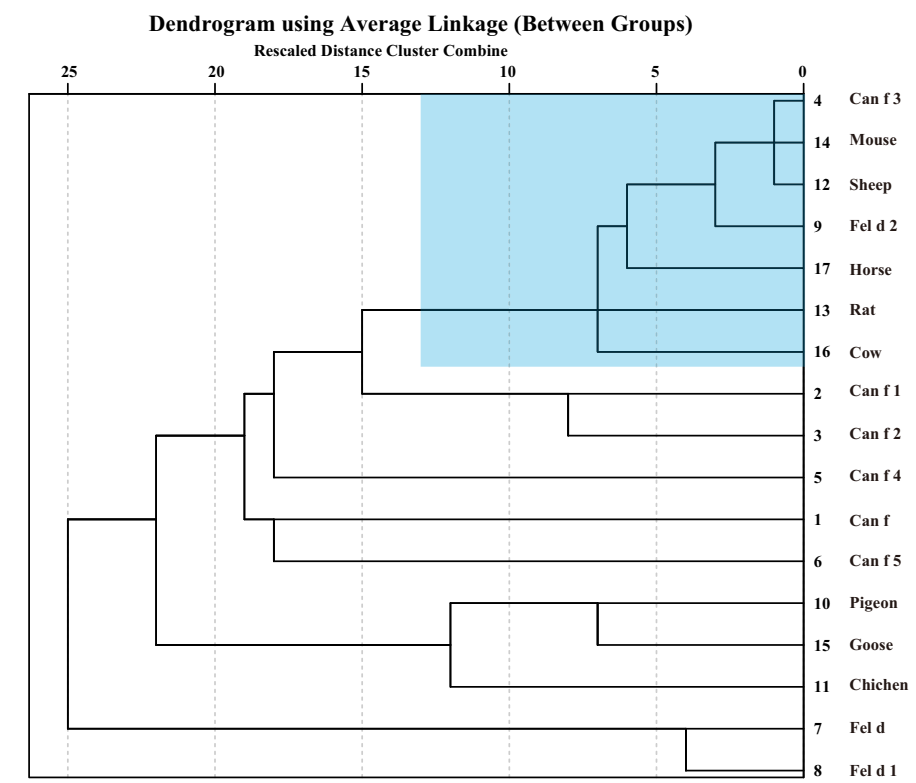

A

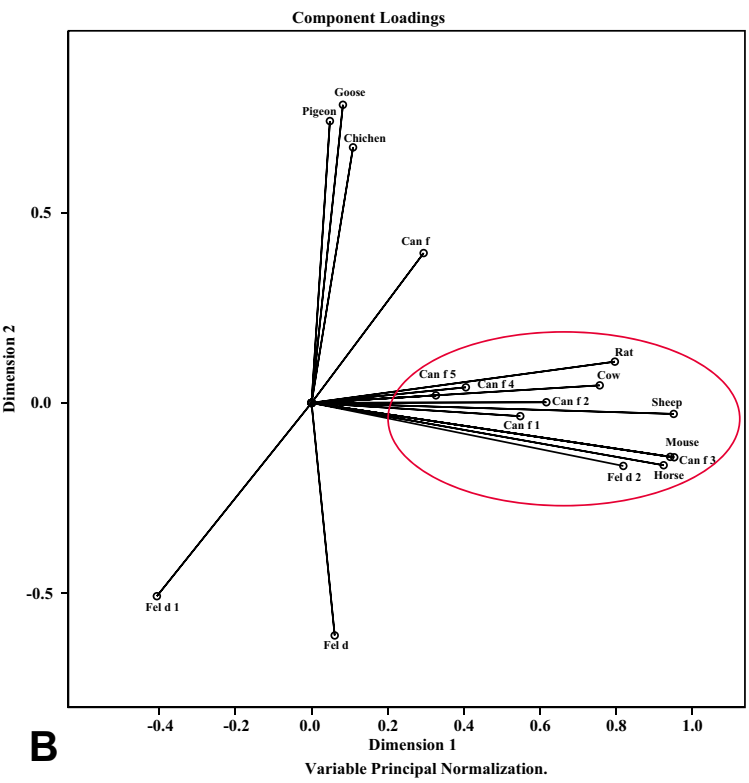

Figure 3 Hierarchical cluster (A) and optimal scale analysis (B) of the connection between components and furry animal crude extracts. Hierarchical clustering analysis, also known as hierarchical cluster analysis, is an algorithm that organizes similar objects into groups called clusters. Optimal scale analysis is a 'dimensional reduction' analytical method. Using specific immunoglobulin E levels as categorical variables, the closer the two points of the allergens were, the higher the correlation between the two factors. Abbreviations: Can f, Canis familiaris; Fel d, Felis domesticus.

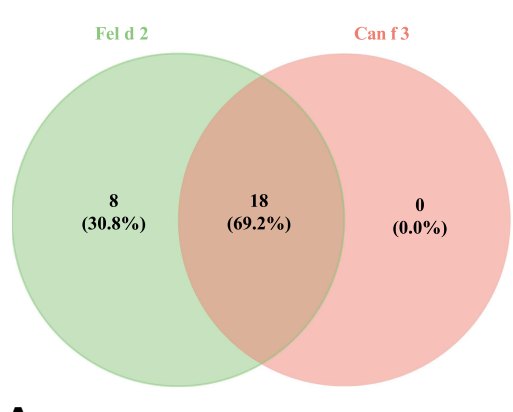

A

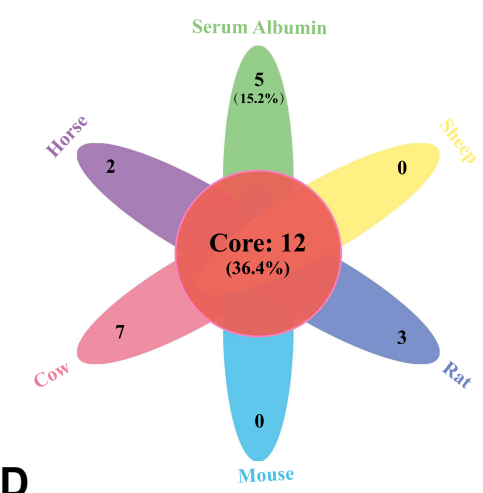

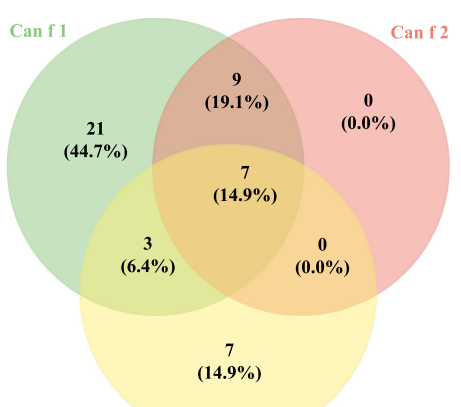

B

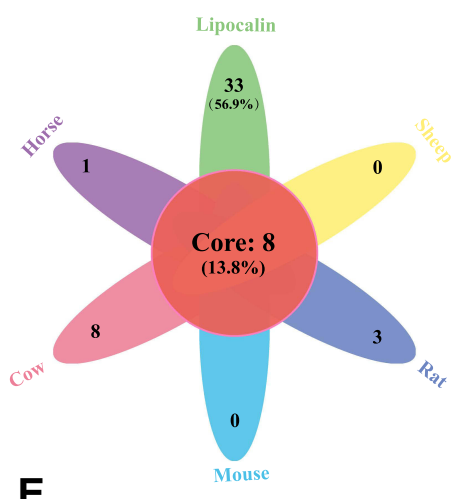

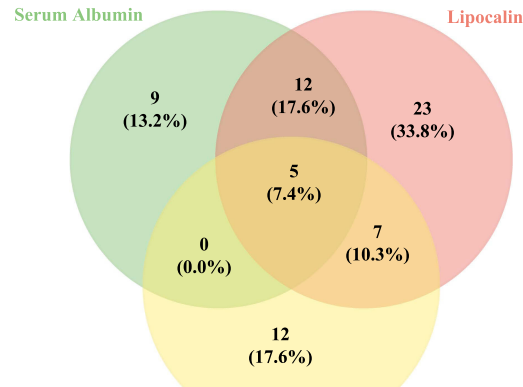

C

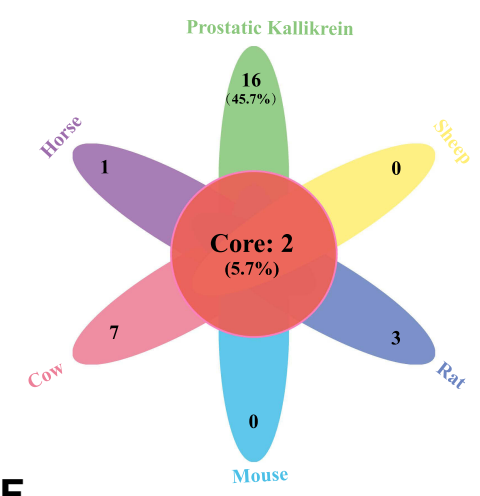

Figure 4 Plot Venn diagrams for serum albumin (A), lipocalin (B), among the three proteins $(\mathbf{C})$, and three proteins link to the extracts from five furry animals (D-F). Core represents the number and proportion of cases where all components are positive. 


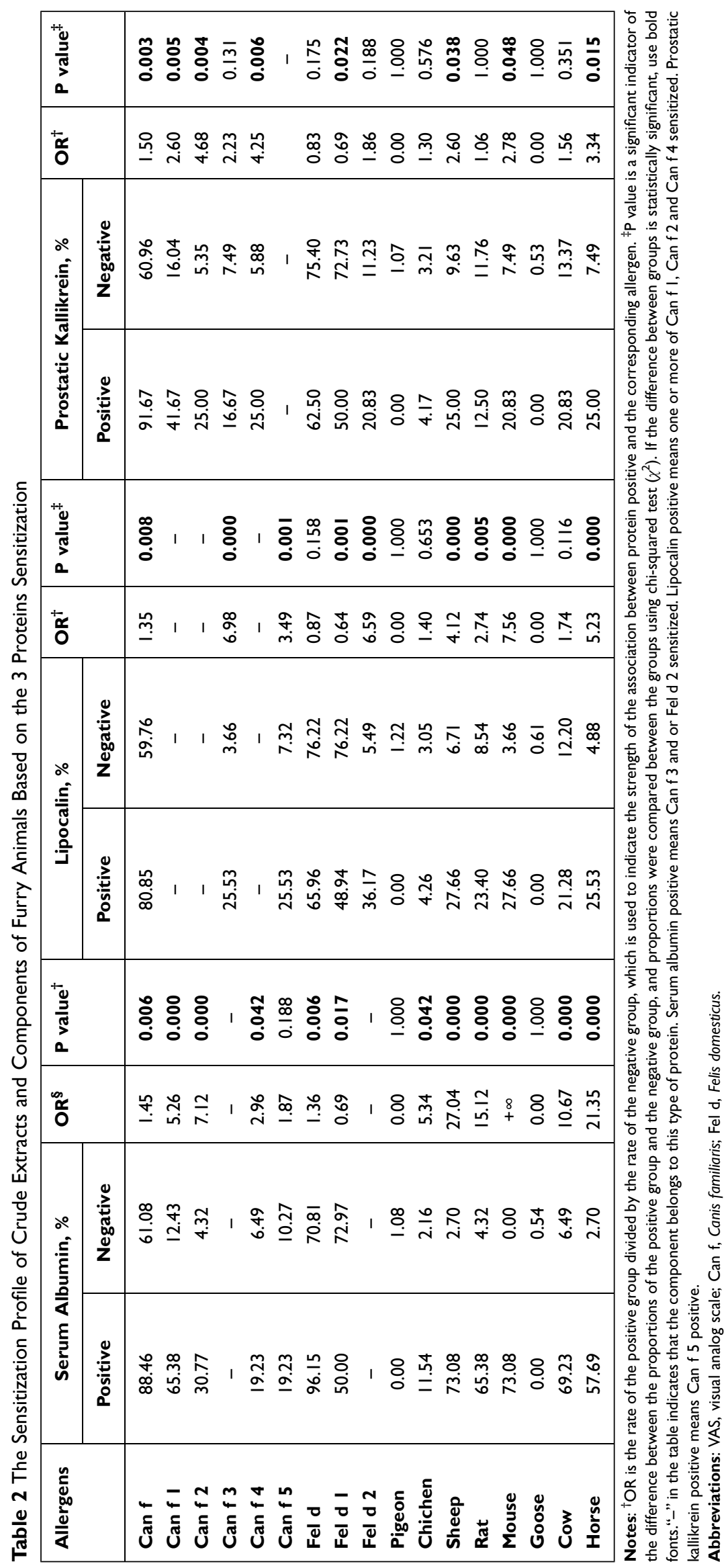



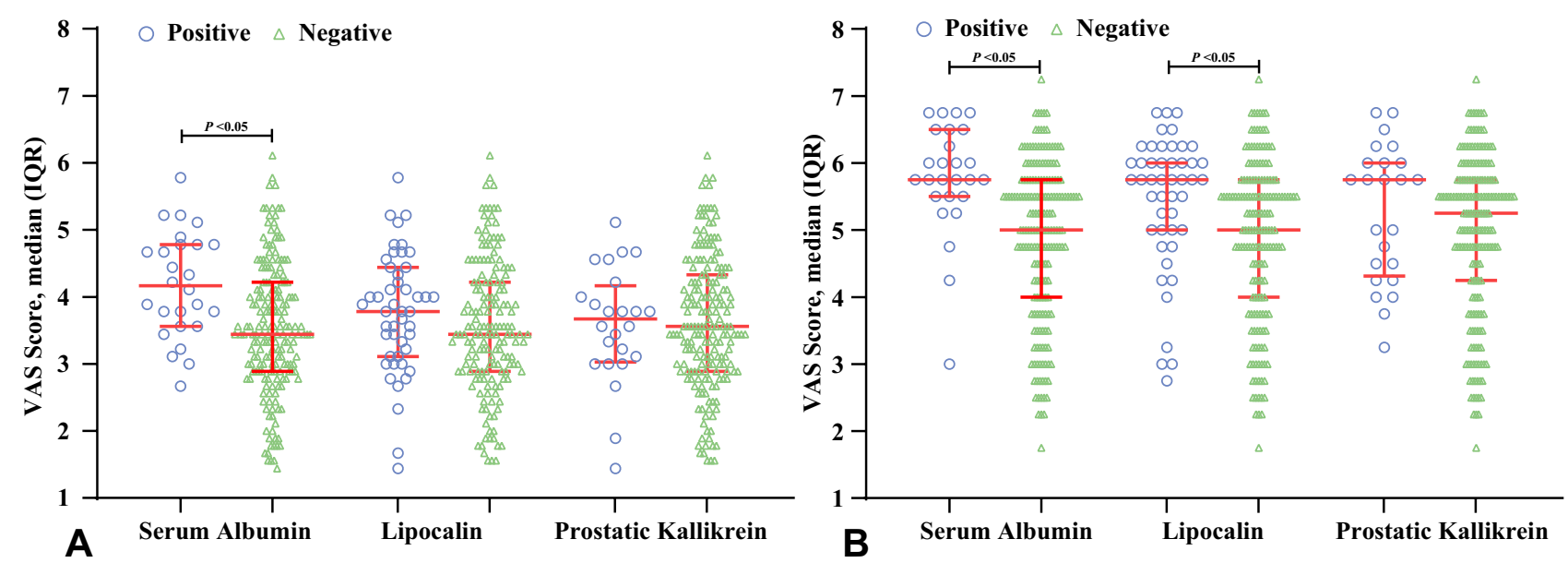

Figure 5(A, B) Differences in VAS scores for symptoms and quality of life in patients with rhinitis. VAS- visual analog scale for serum albumin positive patients means Can $\mathrm{f} 3$ and/or Fel $\mathrm{d} 2$ sensitized. Positive lipocalin indicates sensitization to one or more of Can $\mathrm{f} I$, Can $\mathrm{f} 2$ and Can $\mathrm{f} 4$ allergens. Prostatic kallikrein positivity is defined as Can f 5 positivity. Mann-Whitney $U$ test was used to compare the distribution difference between the two groups, and $P<0.05$ was considered statistically significant.

especially cats and dogs, affect $7 \%$ to $25 \%$ of allergy patients. $^{23}$ Further research on allergic diseases caused by furry animals is of great importance for the clinical prevention, diagnosis and treatment of allergies. In this study, 211 patients with allergic rhinitis sensitized to cats and/or dogs were recruited to analyze the sensitization profiles and clinical associations of various furry animal crude extracts and components based on CRD.

We observed that the positive rate of SA and 5 furry animals (including cow, sheep, rat, mouse, horse and chicken) in double-sensitized patients were significantly higher than that in dog- or cat-sensitized only patients, suggesting that double-sensitized to cat and dog and multiple sensitized to uncommon animals were closely related to SA, so we speculated that SA may play a significant role as cross-reacting allergens in individuals sensitized to dander of multiple animal species, which was consistent with previous studies. ${ }^{24,25}$

The hierarchical cluster and optimal scale analysis results showed that there were significant correlations between components (Can f 1-5 and Fel d 2) and the 5 furry animals (mouse, sheep, Horse, rat, cow), especially between SA (Can f 3, Fel d 2) and the 5 furry animals. Guinea pig SA has been shown to be highly homologous in protein sequence identity (70-75\%) compared to other mammalian pet SA and exhibits a strong IgE crossreaction in the SA family. ${ }^{15}$ Feline SA (Fel d 2) is best known for its cross-reactivity with porcine albumin and its involvement in pork-cat syndrome. ${ }^{26}$ The first studies from France highlighted the possible occurrence of pork allergy in individuals sensitized to cat dander (the so-called pork- cat syndrome), ${ }^{27}$ and the potential cross-reactivity between cat and pig SAs was confirmed years later. ${ }^{28}$

Both Can f 3 and Fel d 2 belong to the SA family, and the Venn diagram showed a high degree of overlap (nearly $70 \%$ ). Can $\mathrm{f} 1,2$ and 4 all represent lipocalins but sequence identities between them are very low $(<30 \%)$. Each of them actually represents a completely independent allergen. ${ }^{1}=$. Meanwhile, there was only a slight overlap among SA, lipocalin and prostatic kallikrein. The results of the Venn diagram also indicated that SA had a strong potential cross-reaction with mouse, sheep, horse, rat and cow due to considerable overlap for $\operatorname{IgE}$ binding to SA, with $48.5 \%$ of sera reacting to one or more of 5 furry animals, and $36.4 \%$ were cross-sensitized by SA and all 5 furry animals. Lipocalin and prostatic kallikrein, on the other hand, had only $13.8 \%$ and $5.7 \%$ overlapped with the 5 furry animals. SAs represent a group of minor allergens in mammals. In some cases, they have been well identified (including Fel d 2 in cats, Can f 3 in dogs, Equ c 3 in horses), but SAs have also been found to be sensitizing agents in rats, mice, and rabbits. There is a wide range of cross-reactions between SA from different furry animals. ${ }^{29}$ Prostatic kallikrein Can $\mathrm{f} 5$ is an androgen-regulated protein expressed in the prostate and hence is present only in male dogs, and there is no strong cross-reactivity between Can $\mathrm{f} 5$ and the extracts from other furry animals. ${ }^{24,30}$ Lipocalins, as an important sensitizing protein in animals, can often predict the development of $\operatorname{IgE}$ cross reactions based on sequence identity, ${ }^{29}$ Previous studies have also shown that cross-reactivity occurs only between those lipocalins with a high degree of homology. ${ }^{11,31}$ However, 
since only a limited panel of lipocalin components of cat were detected in this study, and no more lipocalin components of other furry animals were commercially available for detection, it may lead to the differences between the results of lipocalin in this study and previous studies. Liccardi et al pointed out that the risk of developing sensitization to furry animals is higher in cat/dog sensitized individuals. Specifically, patients sensitized to cat/ dog allergens have a 14-fold higher risk of developing sensitization to other furry animals, such as horses, rabbits, rats, mice, guinea pigs, hamsters, and cows. ${ }^{6}$ In this study, the sensitization rate of most of the animal crude extracts and components in patients who were SA-positive was significantly higher than that of the patients who were SAnegative. Especially for mouse, sheep, horse, rat, and cow, which OR values were more than 10 . Studies have shown that there is a significant cross-reaction between SAs in cats, dogs, horses and pigs, ${ }^{28,32}$ and our research further extends these to furry animals. On the other hand, higher positive rates of other furry animals extracts were also observed in patients with lipocalin-sensitized, but it is necessary to detect the lipocalin components of more other animals to determine whether it is caused by lipocalin cross reaction.

Among the 211 AR patients enrolled in this study, the proportion of asthma was between $60 \%$ and $80 \%$, regardless of whether patients had sensitives to SA, lipocalin and prostatic kallikrein, and there was no significant difference between the positive group and the negative group. A pediatric population-based study from northern Sweden reported that sensitizations to SA was uncommon and not associated with asthma. ${ }^{33}$ However, more studies support that sensitization to furry animals is associated with severe and persistent respiratory diseases, especially in the case of sensitizations to multiple animal components. ${ }^{20,33}$ Therefore, we further analyzed the symptoms and LoQ scores of patients with allergic rhinitis. A study showed that sensitizations to cat, dog and horse SA were associated with more severe respiratory symptoms, ${ }^{34}$ and it may be speculated that type- 2 inflammation could be activated by the exposure to furry animal SA. Sensitizations to lipocalins have been shown to be related to airway hyperresponsiveness. ${ }^{7}$ The VAS of symptoms and LoQ in the SA-sensitized patients was higher than that in unsensitized patients, and patients with lipocalin-sensitivities had a worse LoQ.

One of the limitations of this study is that there are no other commercially available sIgE reagents for rare furry animals. In addition, the allergic rhinitis patients recruited in this study were sensitized to cats and/or dogs, and it could not be confirmed that the patients were truly allergic to cats or dogs because the ethics committee of our unit could not ethically approve animal allergen challenge tests in patients. The study also did not collect some important clinical information in patients, such as pulmonary function indicators, FeNO, eosinophils and so on, which would have allowed us to better explain how SA causes more severe symptoms.

In conclusion, Serum albumin Fel d 2 and Can $\mathrm{f} \mathrm{3}$, as minor allergens in cats and dogs, but not lipocalin or prostatic kallikrein, is associated with other furry animals presumably due to serum albumin cross-reactivity. Patients sensitized with serum albumin had a significantly higher risk of sensitization to other animals and had a higher rhinitis VAS score.

\section{Abbreviations}

AR, allergy rhinitis; AS, asthma; AR \& AS, AR combined with AS; CRD, component resolved diagnosis; IUIS, International Union of Immunological Societies; SA, serum albumin; PK, prostatic kallikrein; VAS, visual analogue scale; LoQ, life of quality; sIgE, specific immunoglobulin E; ARIA, Allergic Rhinitis and its Impact on Asthma; GINA, Global Initiative for Asthma guidelines; Can f, Canis familiaris; Fel d, Felis domesticus; IU/mL, international unit per milliliter.

\section{Statement of Ethics}

This study and the use of the human serum samples were approved by the Ethics Committee of the First Affiliated Hospital of Guangzhou Medical University (GYYY-201673). It was conducted in accordance with the Declaration of Helsinki. Written informed consent was provided from all adult patients or legal guardian of participants under 18 years of age.

\section{Acknowledgments}

This study was supported by the National Natural Science Foundation of China (NSFC 81871736), Guangdong Provincial Medical Research Foundation (A2019224) and Guangzhou Science and Technology Foundation (201804020043).

\section{Author Contributions}

All authors contributed to data analysis, drafting or revising the article, gave final approval of the version to be published, agreed to the submitted journal, and agree to be accountable for all aspects of the work. 


\section{Disclosure}

There are no financial or other issues that might lead to conflict of interest.

\section{References}

1. Hemmer W, Sestak-Greinecker G, Braunsteiner T, Wantke F, Wöhrl S. Molecular sensitization patterns in animal allergy: relationship with clinical relevance and pet ownership. Allergy. 2021. doi:10.1111/all.14885

2. Bjerg A, Winberg A, Berthold M, Mattsson L, Borres MP, Ronmark E. A population-based study of animal component sensitization, asthma, and rhinitis in schoolchildren. Pediatr Allergy Immunol. 2015;26:557-563. doi:10.1111/pai.12422

3. Gergen PJ, Mitchell HE, Calatroni A, et al. Sensitization and exposure to pets: the effect on asthma morbidity in the US population. J Allergy Clin Immunol Pract. 2018;6:101-7 e2. doi:10.1016/j.jaip.2017.05.019

4. Nilsson OB, van Hage M, Gronlund H. Mammalian-derived respiratory allergens - implications for diagnosis and therapy of individuals allergic to furry animals. Methods. 2014;66:86-95. doi:10.1016/j. ymeth.2013.09.002

5. Man's best friend: global pet ownership and feeding trends. Available from: https://www.gfk.com/insights/mans-best-friend-global-petownership-and-feeding-trends. Accessed October 11, 2021.

6. Liccardi G, Passalacqua G, Salzillo A, et al. Is sensitization to furry animals an independent allergic phenotype in nonoccupationally exposed individuals? J Investig Allergol Clin Immunol. 2011;21:137-141.

7. Nordlund B, Konradsen JR, Kull I, et al. IgE antibodies to animal-derived lipocalin, kallikrein and secretoglobin are markers of bronchial inflammation in severe childhood asthma. Allergy 2012;67:661-669. doi:10.1111/j.1398-9995.2012.02797.x

8. Kalayci O. In-vitro diagnosis of allergic diseases. Clin Biochem. 2014;47:728-729. doi:10.1016/j.clinbiochem.2014.05.031

9. Asarnoj A, Hamsten C, Waden K, et al. Sensitization to cat and dog allergen molecules in childhood and prediction of symptoms of cat and dog allergy in adolescence: a BAMSE/MeDALL study. $J$ Allergy Clin Immunol. 2016;137:813-21 e7. doi:10.1016/j.jaci.2015.09.052

10. Madhurantakam C, Nilsson OB, Uchtenhagen H, et al. Crystal structure of the dog lipocalin allergen Can $\mathrm{f} 2$ : implications for cross-reactivity to the cat allergen Fel d 4. $\mathrm{J} \mathrm{Mol}$ Biol. 2010;401:68-83. doi:10.1016/j.jmb.2010.05.043

11. Nilsson OB, Binnmyr J, Zoltowska A, Saarne T, van Hage M, Gronlund $H$. Characterization of the dog lipocalin allergen Can $f$ : the role in cross-reactivity with cat and horse. Allergy. 2012;67:751-757. doi:10.1111/j.1398-9995.2012.02826.x

12. Apostolovic D, Sanchez-Vidaurre S, Waden K, et al. The cat lipocalin Fel $\mathrm{d} 7$ and its cross-reactivity with the dog lipocalin Can f 1. Allergy. 2016;71:1490-1495. doi:10.1111/all.12955

13. Saarelainen S, Rytkonen-Nissinen M, Rouvinen J, et al. Animal-derived lipocalin allergens exhibit immunoglobulin E cross-reactivity. Clin Exp Allergy. 2008;38:374-381. doi:10.1111/j.1365-2222.2007.02895.x

14. Matricardi PM, Kleine-Tebbe J, Hoffmann HJ, et al. EAACI molecular allergology user's guide. Pediatr Allergy Immunol. 2016;27 (Suppl 23):1-250.

15. Spitzauer S, Pandjaitan B, Söregi G, et al. IgE cross-reactivities against albumins in patients allergic to animals. J Allergy Clin Immunol. 1995;96:951-959. doi:10.1016/S0091-6749(95)70233-4

16. Spitzauer S, Schweiger C, Sperr WR, et al. Molecular characterization of dog albumin as a cross-reactive allergen. J Allergy Clin Immunol. 1994;93:614-627. doi:10.1016/S0091-6749(94)70073-7
17. Mattsson L, Lundgren T, Everberg H, Larsson H, Lidholm J. Prostatic kallikrein: a new major dog allergen. J Allergy Clin Immunol. 2009;123:362-368. doi:10.1016/j.jaci.2008.11.021

18. Goubran Botros H, Gregoire C, Rabillon J, David B, Dandeu JP. Cross-antigenicity of horse serum albumin with dog and cat albumins: study of three short peptides with significant inhibitory activity towards specific human $\mathrm{IgE}$ and $\mathrm{IgG}$ antibodies. Immunology. 1996;88:340-347. doi:10.1046/j.1365-2567.1996.d01-669.x

19. Vicente-Serrano J, Caballero ML, Rodriguez-Perez R, et al. Sensitization to serum albumins in children allergic to cow's milk and epithelia. Pediatr Allergy Immunol. 2007;18:503-507. doi:10.1111/j.1399-3038.2007.00548.x

20. Tsolakis N, Malinovschi A, Nordvall L, et al. Sensitization to minor cat allergen components is associated with type-2 biomarkers in young asthmatics. Clin Exp Allergy. 2018;48:1186-1194. doi:10.1111/cea.13135

21. Brożek JL, Bousquet J, Agache I, et al. Allergic Rhinitis and its Impact on Asthma (ARIA) guidelines-2016 revision. J Allergy Clin Immunol. 2017;140:950-958. doi:10.1016/j.jaci.2017.03.050

22. Spector SL, Nicklas RA, Chapman JA, et al. Symptom severity assessment of allergic rhinitis: part 1. Ann Allerg Asthma Immunol. 2003;91:105-114. doi:10.1016/S1081-1206(10)62160-6

23. Satyaraj E, Wedner HJ, Bousquet J. Keep the cat, change the care pathway: a transformational approach to managing Fel d 1, the major cat. Allergen Allerg. 2019;74(Suppl 107):5-17. doi:10.1111/all.14013

24. Liccardi G, Asero R, D'Amato M, D'Amato G. Role of sensitization to mammalian serum albumin in allergic disease. Curr Allergy Asthma Rep. 2011;11(5):421-426. doi:10.1007/s11882-011-0214-7

25. Schoos AM, Nwaru BI, Borres MP. Component-resolved diagnostics in pet allergy: current perspectives and future directions. J Allergy Clin Immunol. 2021;147(4):1164-1173. doi:10.1016/j.jaci.2020.12.640

26. Konradsen JR, Fujisawa T, Hage MV, et al. Allergy to furry animals: new insights, diagnostic approaches, and challenges. J Allerg Clin Immunol. 2015;135:616-625. doi:10.1016/j.jaci.2014.08.026

27. Drouet M, Boutet S, Lauret MG, et al. The pork-cat syndrome or crossed allergy between pork meat and cat epithelia. I Allergol Immunol. 1994;26:166-168

28. Hilger C, Kohnen M, Grigioni F, Lehners C, Hentges F. Allergic cross-reactions between cat and pig serum albumin. Study at the protein and DNA levels. Allergy. 1997;52:179-187. doi:10.1111/ j.1398-9995.1997.tb00972.x

29. Suzuki S, Nwaru BI, Ekerljung L, et al. Characterization of sensitization to furry animal allergen components in an adult population. Clin Exp Allergy. 2019;49:495-505. doi:10.1111/cea.13355

30. Ukleja-Sokołowska N, Lis K, Żbikowska-gotz M, Adamczak R, Bartuzi Z. Analysis of allergen profile in patients sensitized to canine allergen and potential Can f 5 cross-reactivity with human PSA. Int J Immunopathol Pharmacol. 2021;35:20587384211023670. doi:10.1177/20587384211023670

31. Hilger C, Swiontek K, Arumugam K, Lehners C, Hentges F. Identification of a new major dog allergen highly cross-reactive with Fel d 4 in a population of cat- and dog-sensitized patients. J Allergy Clin Immunol. 2012;129(4):1149-1151. doi:10.1016/j. jaci.2011.10.017

32. Cabañas R, López-Serrano MC, Carreira J, et al. Importance of albumin in cross-reactivity among cat, dog and horse allergens. J Investig Allergol Clin Immunol. 2000;10:71-77.

33. Konradsen JR, Nordlund B, Onell A, Borres MP, Grönlund H, Hedlin G. Severe childhood asthma and allergy to furry animals: refined assessment using molecular-based allergy diagnostics. Pediatr Allergy Immunol. 2014;25:187-192. doi:10.1111/pai.12198

34. Uriarte SA, Sastre J. Clinical relevance of molecular diagnosis in pet allergy. Allergy. 2016;71:1066-1068. doi:10.1111/all.12917 


\section{Publish your work in this journal}

The Journal of Asthma and Allergy is an international, peer-reviewed open-access journal publishing original research, reports, editorials and commentaries on the following topics: Asthma; Pulmonary physiology; Asthma related clinical health; Clinical immunology and the immunological basis of disease; Pharmacological interventions and

Submit your manuscript here: https://www.dovepress.com/journal-of-asthma-and-allergy-journal new therapies. The manuscript management system is completely online and includes a very quick and fair peer-review system, which is all easy to use. Visit http://www.dovepress.com/testimonials.php to read real quotes from published authors. 